\title{
Digitalización, monitorización y evaluación del impacto de la Economía Social. Análisis en el Tercer Sector de Acción Social español: el caso de Juntos por el Empleo
}

\section{Mercedes Valcárcel-Dueñas y Marta Solórzano-García}

RESUMEN: Durante los últimos años se han elaborado numerosos estudios sobre la necesidad de que las entidades del Tercer Sector de Acción Social (TSAS) desarrollen habilidades para comunicar y demostrar sus resultados y la eficiencia en el desempeño de su actividad. En este proceso, la monitorización y la evaluación se convierten en actividades claves para hacer partícipes a los distintos grupos de interés del impacto por ellas generado. Junto a esto, la sociedad ha vivido una evolución digital que ha generado cambios profundos en todos los ámbitos, grupos e individuos.

Partiendo de un análisis realizado desde la teoría institucional y la teoría de la dependencia de recursos para poner en valor la importancia que adquiere este proceso dentro del contexto de los requerimientos de los financiadores y de los grupos de interés en general, planteamos la necesidad de identificar las nuevas herramientas digitales y formas de trabajo con las mismas que pueden apoyar la realización de la evaluación en las entidades del TSAS y que ésta sea de utilidad para el cumplimiento de la misión y los objetivos de las organizaciones. Analizamos el caso de Juntos por el Empleo, iniciativa promovida por la Fundacion Accenture junto a varias organizaciones sociales, empresas y administraciones públicas, cómo práctica de interés en la implementación de nuevas herramientas digitales que han facilitado el trabajo compartido de gestión y evaluación de sus proyectos sociales. El enfoque de impacto colectivo se descubre como una nueva forma, colaborativa y estructurada, necesaria para abordar los problemas a los que se enfrenta el TSAS.

PALABRAS CLAVE: Tercer sector de acción social, digitalización, monitorización, evaluación del impacto social, teoría institucional, teoría de la dependencia de recursos.

CLAVES ECONLIT: A13, B52, D63, L14, L31, 033.

Cómo citar este artículo / How to cite this article: VALCÁRCEL-DUEÑAS, M. \& SOLÓRZANO-GARCÍA, M. (2019): "Digitalización, monitorización y evaluación del impacto de la Economía Social. Análisis en el Tercer Sector de Acción Social español: el caso de Juntos por el Empleo", CIRIEC-España, Revista de Economía Pública, Social y Cooperativa, 95, 143-159, DOI: 10.7203/CIRIEC-E.95.13128.

Correspondencia: Marta Solórzano-García (correspondencia), Profesora Titular de Universidad, Departamento de Organización de Empresas, Universidad Nacional de Educación a Distancia, UNED, msolorzano@cee.uned.es; Mercedes Valcárcel-Dueñas, Directora general de Fundación Tomillo, Universidad de Córdoba. 


\section{EXPANDED ABSTRACT}

\section{Digitalization, monitoring and evaluation of the social economy impact. Analysis of spanish third sector of social action. "Juntos por el empleo" case study}

In recent years, numerous studies have been conducted assessing the needs of Third Sector Social Action (TSAS from now on) entities to develop skills that enable them to communicate and demonstrate their results and efficiency.

In this process, monitoring, understood as the collection of information on aspects related to its management and the tracking of its progress in a routine and systematic way, and evaluation, understood as the collection of data to make judgments with the consequent assessment, become key activities to make the various stakeholders participate in the impact that they generate.

TSAS entities' stakeholders are strongly demanding information about their performance and results, information that can reflect a holistic picture of the socio-economic impact they are really generating. These requirements are exhorted either formally or directly through evaluation exigencies included in the contracts or either indirectly through norms that social organizations have to comply with. Therefore, TSAS entities are conditioned by the influence of logics and norms that other groups uphold.

A standardized monitoring and evaluation methodology that integrates, with a common and understandable language, internal information needs with external stakeholders information demands is needed. One of the groups that have heavily exerted pressure on the former is of the funders, so that TSAS organizations need to communicate and report what they do and how they do it in order to receive financing.

This demonstration of the value generated require metrics that are based on the developing of indicators that social impact measurement incorporates. It is not enough to show the information that favours the organization or project that is meant to communicate but as a result of institutional pressures exercised over the TSAS, the level of transparency on its performance is very high. Along with this, society has experienced a digital evolution that has generated profound changes in every area, group and individual.

In this paper, we will focus on the changes that affect monitoring and evaluation practices of TSAS entities. An analysis from the theory is needed not only to build an evidence base about 
monitoring and evaluation practice and its benefits, if any, but also to place that practice within the context of funders' requirements and evaluation activity.

In order to analyse the pressure of the funders, we will rely on the institutional and on the resources dependence theories, which provides the basis for studying how external pressures affect organizational behaviour. According to institutional theory, the information provided by entities about what they do and how they do it is a consequence of the demands placed upon them by their stakeholders: consumers, investors, employees, public agents, funders and others. These demands constitute a way of institutional pressure that motivates organizations to "improve" the information they provide. And the situation of dependence on the funders of the TSSA entities means that they are forced to comply with the demands imposed on the information to be communicated and on the actions and results reporting.

In parallel, in order to meet their own economic, social and environmental objectives, these entities need to have, process and communicate a large amount of information. These data must also answer to specific requirements for facilitating the development of an effective management and for controlling their results and possible deviations.

In this sense, it's required to identify new digital tools and working practices that can support evaluation as well as making these tools and practices to contribute to the entities' mission and objectives. "Juntos por el Empleo", an initiative promoted by Fundación Accenture together with different social entities, business and public organizations, is analysed as an interesting practice for new digital tools implementation that have improve social projects and collective practices monitoring and evaluation.

This paper's main objective is to highlight the TSAS entities dependence on their funders and how this dependence requires them to provide information about their activities and results in a specific format. Within this framework we show "Juntos por el empleo" project as a practice that is being developed to face these requirements by the Spanish TSAS entities.

The work is developed with the following steps: we start from an analysis from the institutional theory and the resources dependence theory to justify the importance of the monitoring and evaluation of the TSAS entities' results to meet the funders and stakeholders' requirements. Next, we discuss the need to identify the new digital tools and ways of working that help these monitoring and evaluating processes, to finish with the case study of "Juntos por el empleo" as a collective impact project focused on the implementation of new digital tools to facilitate the management and evaluation of social activities.

KEYWORDS: Third Sector Social Action, digitalization, monitoring, evaluation of the social impact, institutional theory, resources dependence theory. 


\section{Introducción}

En los últimos años, a las organizaciones del Tercer Sector de Acción Social se les está demandando, cada vez con más fuerza, que reflejen información sobre el impacto social y medioambiental que generan. Los distintos grupos que afectan y a los que les afecta su actividad, entre los que se encuentran inversores, consumidores, financiadores, empleados y administraciones públicas, son conscientes de que para valorar a las entidades del Tercer Sector de Acción Social (TSAS de ahora en adelante) necesitan una información que proporcione una imagen holística de su actividad, no limitada a la que transmiten los indicadores financieros, y han comenzado a presionar a las entidades para que den a conocer información sobre sus resultados sociales y medioambientales (Costa y Pesci, 2016; Ebraim y Rangan, 2014).

Los distintos grupos de interés de las organizaciones del TSAS ejercen este control bien formal y directamente, a través de exigencias de evaluación incluidas en los contratos, o indirectamente, mediante normas que los individuos y organizaciones deben cumplir para lograr la aprobación y verificación de que su actividad cumple los requisitos establecidos (Power, 1999). De forma que las organizaciones del TSAS están condicionadas por la influencia de las lógicas y normas que sostienen otros grupos (Arvison y Lyon, 2014).

Estas demandas han generado un incremento de los informes de sostenibilidad por parte de las organizaciones del TSAS. Ahora bien, ¿se corresponde este aumento con una mejora en la transparencia de las entidades del TSAS? Según han concluido investigaciones previas, el contenido y profundidad de los informes de sostenibilidad varía significativamente entre entidades (Higgins, Stubbs y Milne, 2018; Ehnert, et al., 2016; Lock y Seele, 2016; Kolk, 2004). Incluso existen estudios que reflejan la falta de calidad en estos informes (Morhard, 2010; Milne, Tregidga y Walton, 2009; Cooper y Owen, 2007; Sinclair y Walton, 2003).

Es necesaria una metodología estandarizada de monitorización y evaluación que integre, con un lenguaje común y comprensible, las necesidades internas de información, para el aprendizaje y desarrollo de la organización, con la información que les exigen los grupos de interés externos para rendir cuentas de sus resultados (Maas, Schaltegger y Crutzen, 2016; Vigneau, Humphreys y Moon, 2015; Bebbington, Brown, y Frame, 2007).

Uno de los grupos externos que mayor presión ejerce es el de los financiadores (Arvidson y Lyon, 2014), de forma que para poder obtener los recursos necesarios para desarrollar su actividad, las organizaciones del TSAS necesitan comunicar y rendir cuentas de lo que hacen y de cómo lo hacen (Moxham, 2010; Nicholls, 2010). Esta demostración del valor generado exige unas métricas que se 
basan en la elaboración de indicadores que incorpora la medición. No es suficiente con mostrar aquella información que es favorable a la organización o al proyecto que se quiere comunicar, sino que, fruto de las presiones institucionales que se ejercen sobre el tercer sector, la exigencia de transparencia en el desempeño es muy alta (Nicholls, 2010; Ebrahim \& Weisband, 2007; Gibelman y Gelman, 2004).

De forma paralela, y a nivel interno, estas entidades, para poder cumplir con los objetivos económicos, sociales y medioambientales que se le exigen, precisan disponer, procesar y comunicar una gran cantidad de información, que además debe responder a unos requisitos específicos, tanto para poder desarrollar una gestión eficaz, como para poder controlar los resultados y posibles desviaciones.

El objetivo principal de este artículo es poner en relieve la dependencia de las entidades del TSAS de sus principales proveedores de recursos financieros y cómo esa dependencia les exige proporcionarles la información sobre sus actividades y resultados en un formato determinado. Ofrecemos, asimismo, el caso de Juntos por el Empleo como práctica que se está desarrollando para enfrentar estos requisitos por parte de las entidades del TSAS españolas.

Para analizar la presión de los financiadores nos apoyaremos en la teoría institucional y la de la dependencia de recursos, que proporciona la base para estudiar cómo afectan las presiones externas al comportamiento organizativo. De acuerdo con la teoría institucional, el hecho de que las organizaciones proporcionen información sobre lo que hacen y cómo lo hacen es consecuencia de las demandas que sobre ellas ejercen los grupos de interés: consumidores, inversores, empleados, agentes públicos, financiadores y otros. Estas acciones constituyen una forma de presión institucional que motiva que las organizaciones necesiten "mejorar" la información que proporcionan. Por otra parte, el hecho de que las entidades del TSAS se encuentren en una situación de dependencia respecto a los financiadores hace que se vean forzadas a acatar las exigencias que éstos les imponen sobre la información a difundir y la rendición de cuentas sobre sus actuaciones y resultados.

El trabajo se desarrolla de acuerdo a la secuencia siguiente: partimos de un análisis realizado desde la teoría institucional y la teoría de la dependencia de recursos para justificar la importancia de la monitorización y evaluación de los resultados de las entidades del TSAS para atender a los requerimientos de los financiadores y de los grupos de interés en general. A continuación, argumentamos la necesidad de identificar las nuevas herramientas digitales y formas de trabajo que ayuden a los procesos de monitorización y evaluación de resultados de las organizaciones, para terminar con el estudio del caso de Juntos por el Empleo, cómo práctica intersectorial de impacto colectivo de interés para el estudio de la implementación de nuevas herramientas digitales que han facilitado el trabajo compartido de gestión y evaluación de proyectos de impacto social. 


\section{Análisis de las demandas de los financiadores desde el marco de la teoría institucional y teoría de la dependencia de recursos}

La teoría institucional nos proporciona una base para estudiar cómo afectan las presiones externas al comportamiento organizativo. En el caso que nos ocupa, la racionalidad subyacente en la recogida y transmisión de información por las entidades del TSAS sobre los aspectos relacionados con su gestión y resultados es hacer frente a las exigencias de sus grupos de interés. Éstas constituyen una forma de presión institucional que puede motivar a las organizaciones a tratar de mejorar los métodos y herramientas con los que recopilar información sobre su actividad y con los que medir sus resultados económicos, sociales y medioambientales.

De acuerdo con la teoría institucional, a medida que las prácticas organizativas se institucionalizan, la sociedad empieza a considerarlas legítimas y son adoptadas por las organizaciones, buscando la legitimación y no por razones de eficiencia (Meyer y Rowan, 1977; DiMaggio y Powell, 1983; Zucker, 1987). Las organizaciones adoptan estructuras, programas, políticas y procedimientos cada vez más homogéneos dentro de su propio campo. Este "isomorfismo institucional", como lo denominan DiMaggio \& Powell (1983) ocurre a través de procesos coercitivos, miméticos y normativos (DiMaggio \& Powell, 1983; Meyer \& Rowan, 1977; Scott, 1987). El isomorfismo coercitivo tiene su origen en las presiones políticas y en el problema de la legitimidad, el isomorfismo mimético es el resultado de respuestas estandarizadas a la incertidumbre y el isomorfismo normativo, asociado a la profesionalización (DiMaggio \& Powell, 1983, p. 150)

El isomorfismo coercitivo es fruto de presiones formales e informales impuestas por unas organizaciones a otras que se encuentran en posición de dependencia respecto de las primeras y de las expectativas culturales de la sociedad en la que las organizaciones operan. Las presiones coercitivas proceden de relaciones de poder y política y generalmente se materializan en imposiciones por parte del Estado o de otras organizaciones para que organizaciones con las que mantienen relaciones de dependencia adopten unas estructuras o prácticas determinadas so pena de ser sancionadas. Estas presiones pueden tener también su origen en la dependencia de recursos. La teoría de la dependencia de los recursos se fundamenta en que "la clave de la supervivencia organizativa es la habilidad para adquirir y mantener recursos" (Pfeffer y Salancik, 1978, p. 2). Su hipótesis principal es que ninguna organización es capaz de generar todos los recursos que necesita, por lo que necesitan de su entorno para conseguir parte de los recursos necesarios para su funcionamiento, lo que les sitúa en una situación de dependencia con respecto a las otras organizaciones que controlan los recursos que necesitan (Pfeffer y Salancik, 1978) y este grado de dependencia es función de la importancia y concentración de dichos recursos (Froelich, 1999). Por consiguiente, las organizaciones no son entidades totalmente autónomas que establecen sus objetivos y cómo alcanzarlos de acuerdo con sus propios criterios, sino que se hallan forzadas a acatar los requerimientos de los proveedo- 
res de recursos estratégicos para poder afrontar la incertidumbre y precariedad de su entorno (Froelich, 1999).

Dado que el grado de dependencia de un recurso específico determina la vulnerabilidad de la organización respecto a los impactos externos que generan otros actores del entorno, las entidades del TSAS, que dependen en alta medida de la financiación externa, son organizaciones muy vulnerables (Verbruggen, Christiaens, y Milis, 2011; Bies, 2010;). Por lo tanto, y sobre la base de la teoría de la dependencia de los recursos, cuanto mayor sea la dependencia de una entidad del TSAS de un financiador o grupo de financiadores, más control tendrán éstos en su operativa y sobre su supervivencia y mayor peso tendrá su opinión en las decisiones organizativas.

La necesidad de conseguir recursos financieros hace que las entidades del TSAS estén sometidas a fuertes presiones de sus financiadores. Estas presiones se materializan, entre otras formas, en las exigencias que les imponen para realizar y presentar informes de medición de impacto (Nicholls, 2010). La literatura académica sobre medición de impacto refleja la existencia de relaciones de poder asimétricas, siendo los financiadores los grupos de interés con mayor influencia (Arvidson y Lyon, 2014) y ponen de manifiesto que la razón principal por la que las entidades realizan mediciones de impacto es para obtener recursos (Nguyen, Szkudlarek y Seymour, 2015; Moxham, 2010; Nicholls, 2010). Son los financiadores los que imponen las mediciones de impacto y cómo realizarlas (Dhanani y Connolly, 2012; Eckerd y Moulton, 2011; Moxham, 2010; Ebrahim, 2003, 2005).

\section{El potencial de las tecnologías digitales para demostrar la creación de valor por las ONG}

Quienes toman las decisiones en las entidades del TSAS son conscientes de la necesidad de enfoques de evaluación de impacto que permitan simplificar su elaboración y sistematizar la ejecución de las actividades que su realización entraña.

La medición de impacto necesita definir la información a recopilar para poder construir los indicadores adecuados y medirlos, lo que requiere de modelos conceptuales robustos, como las colecciones de indicadores, y de técnicas analíticas de integración, para poder dotar de significado a los datos cuantitativos y cualitativos recogidos para la medición. Esta constitución de colecciones de indicadores y recogida y análisis de datos necesita ser un proceso transparente, pues los grupos de interés exigen de transparencia a las entidades del TSAS para dotarlas de legitimidad (Gálvez, Caba y López, 2012; Crespy y Miller, 2011; Suchman, 1995).

Scholte (2004) se refiere a la legitimidad por los resultados y mantiene que se refuerza cuando las entidades del TSAS rinden cuentas ante sus grupos de interés utilizando estructuras de toma de deci- 
siones y comunicación transparentes. La rendición de cuentas por las entidades del tercer sector pasa por la generación de cuentas transparentes y para ello precisa de personas con los conocimientos técnicos necesarios y de procesos de monitorización, gestión y medición del valor generado para poder ofrecer a los financiadores la información que demandan. Para poder proveer esta información de acuerdo con los criterios que actualmente exigen los financiadores, las entidades del TSAS deben actuar como organizaciones con un alto grado de profesionalización y estructuradas en torno a tecnologías de la información y la comunicación (Edwards y Hulme, 1995; Ebrahim, 2003).

Simplificar los procesos de rendición de cuentas y dotarlos de transparencia es posible mediante el empleo de tecnología computacional digital basada en la información (Seele, 2016). Gomes (2009) propugna el desarrollo de un nuevo campo, al que denomina "sostenibilidad computacional" que genere nuevos modelos, métodos y herramientas digitales para contribuir a equilibrar las necesidades medioambientales, sociales y económicas para lograr un futuro sostenible. En la misma línea se manifiesta Helbing (2012) cuando propone desarrollar una nueva tecnología de información y comunicación que aborde cuestiones de sostenibilidad.

Seele (2016) defiende la utilidad del "big data" como herramienta para producir datos entre todos los actores involucrados en el tema. Asimismo, existen entidades que han desarrollado nuevos métodos para medir y divulgar informes de sostenibilidad, guías para la estandarización de dichos informes (Knebel y Seele, 2015) y de los indicadores utilizados en las guías de inversión ética (Jensen y Seele, 2013).

En los últimos años, la rápida difusión de tecnologías digitales entre las organizaciones del tercer sector ha traído consigo un gran potencial para demostrar y promocionar la rendición de cuentas de estas entidades frente a sus financiadores y demás grupos de interés (Saxton y Guo, 2011)

Para las entidades del TSAS, internet representa una oportunidad muy rentable para construir y mejorar las relaciones con sus grupos de interés (Hart, 2002). Gandía (2011) evidencia la relevancia de la tecnología para la mejora de la rendición de cuentas y transparencia de las organizaciones no lucrativas y Vaccaro y Madsen (2009) afirman que el empleo de las tecnologías de la información y comunicación y, especialmente, las herramientas basadas en internet, es una nueva práctica de transparencia que puede denominarse "transparencia dinámica".

Por lo tanto deben desarrollarse mecanismos innovadores que permitan demostrar a los grupos de interés el valor económico, social y medioambiental que aporta la entidad. Y para ello, ya no basta con mostrar los resultados obtenidos, también es necesario mostrar la forma en la que han sido alcanzados. En este sentido, han surgido diversas iniciativas dirigidas a hacer frente a las exigencias de monitorización y transparencia que los distintos grupos de interés están imponiendo a las entidades del TSAS y que involucran a las entidades y los financiadores para el diseño conjunto de soluciones basadas en herramientas digitales. 


\section{El apoyo a los requerimientos de información a través de la tecnología: Juntos por el Empleo}

Para el desarrollo de la investigación se utiliza como base metodológica el estudio del caso. La metodología de estudio del caso nos permite examinar "un fenómeno contemporáneo dentro de su contexto real" (Yin 1984, p. 23). El objetivo de este estudio es analizar los procesos y resultados de "Juntos por el Empleo", programa que desarrolla una metodología que pretende hacer de la medición de resultados una palanca para la eficiencia, la comunicación y la transparencia de la entidad y permitir al agente interesado evaluar la capacidad de la entidad en el cumplimiento de sus fines y en la generación de valor, para adoptar, en consecuencia, las decisiones oportunas. En este sentido Eisenhardt (1989, p. 542) concibe un estudio de caso contemporáneo como "una estrategia de investigación dirigida a comprender las dinámicas presentes en contextos singulares". De hecho, el estudio de caso nos permite abordar planteamientos complejos de forma interactiva, abierta y dinámica, así como conocer en mayor profundidad un fenómeno. El estudio de caso es, pues, coherente y pertinente con los objetivos de este estudio al proporcionarnos una información diversa, múltiple y contextualizada.

La significación del caso elegido reside, entre otros factores, en el número de entidades participantes y la variedad de servicios que cubre. Juntos por el Empleo es un proyecto de impacto colectivo en el que participan entidades del TSAS, empresas y administraciones públicas que buscan reducir el desempleo en España, especialmente entre los colectivos más vulnerables. Está coordinado y promovido por la Fundación Accenture y en él participan varias entidades del TSAS y algunos de sus financiadores, todos a través de actividades probono.

Su objetivo es acercar el mundo digital a las entidades del TSAS para generar oportunidades que faciliten el trabajo de los técnicos de empleo y autoempleo y mejorar así sus procesos de acompañamiento y formación. Todo ello con el acompañamiento de empresas que financian y/o apoyan a estas entidades sociales ofreciendo prácticas y trabajo a sus alumnos y de las administraciones públicas responsables de las politicas de empleo.

Esta forma integral y coordinada de abordar el problema social del desempleo de personas vulnerables, incorporando soluciones tecnológicas, permite una mayor eficiencia y eficacia en las actividades de formación para el empleo de las entidades del TSAS y en la inserción laboral de los participantes en los programas. Pero este proyecto colaborativo, dado su alcance, no solo apoya el trabajo de intervención directa de los técnicos de empleo de las entidades sociales. A través de los datos que se incorporan en el sistema y la posibilidad de su gestión, también facilita los procesos de provisión de la información que los financiadores de las entidades del TSAS les exigen para subvencionar sus actividades, su posterior seguimiento y evaluación y la propia gestión y dirección de los programas de intervención de estas entidades por parte de sus responsables. 
Adicionalmente a este eje de digitalización de procesos, se ha promovido una linea de investigación sobre la situación del mercado laboral en España, el Observatorio para el Empleo. Este observatorio tiene una doble vertiente. Por un lado, se nutre de los datos de más de 500.000 personas vulnerables participantes en los programas de empleo de las entidades del TSAS, con más de 74.000 ofertas de trabajo gestionadas y más de 30.000 contratos firmados, proporcionando una información valiosa sobre las perspectivas de creación de empleo y los perfiles más demandados en este colectivo. Por otro lado, basado en análisis estadísticos y en colaboración con FEDEA (Fundación de Estudios de Economía Aplicada), se ha desarrollado una herramienta de perfilado que proporciona un indicador de empleabilidad de los desempleados. La información obtenida con estos estudios permite conocer los colectivos con mayor dificultad para acceder al empleo, compartir esta información con las entidades participantes haciendo sensibilización con las administraciones públicas y facilitar el análisis de las medidas a tomar para apoyar a los que se encuentran en mayor vulnerabilidad. Centrándonos en el dato más significativo, se ha concluido que existen en la actualidad en España cuatro millones de personas vulnerables ante el empleo.

Antes de entrar en el detalle del programa y el impacto que está teniendo en las entidades españolas del TSAS dedicadas a la formación para el empleo, es importante encuadrar el modelo de colaboración y trabajo en red que ha supuesto.

En un mundo en el que los problemas sociales, y en concreto el desempleo, cada vez están condicionados por mayor número de factores y son más complejos, las soluciones para abordarlos requieren actuaciones más integrales y con implicación de mayor número de actores. Así, la colaboración entre entidades sociales se ha convertido en una prioridad en los últimos años.

Esta colaboración no ha estado exenta de dificultades dada la complejidad de integrar en la misma los elementos necesarios para su éxito. ¿Y cuáles son estos elementos? De acuerdo con las conclusiones de los modelos llamados de impacto colectivo, estos elementos son (Kania y Kramer, 2011):

1. La existencia de una agenda conjunta basada en una comprensión común de cómo generar el cambio social a través de las actividades que abordan la resolución de los problemas.

2. El desarrollo de sistemas de medición de impacto compartidos por todas las entidades participantes.

3. La realización de actividades que contribuyan al fin común fijado dentro del ámbito de especialización y la elección de cada una de las entidades.

4. Una comunicación continua que facilite la transparencia y el seguimiento de la agenda común por parte de todos.

5. Una organización u organizaciones de apoyo con personal específico adscrito a la coordinación y gobernanza del programa y un sistema de gobernanza claro y consensuado que facilite la toma de decisiones cuando sea necesario. 
De acuerdo con su objetivo, sus participantes, sus actividades y su forma de gestión Juntos por el Empleo es una iniciativa de impacto colectivo que, mediante el uso de la tecnologia, contribuye a la gestión, la transparencia y la medición de impacto las entidades del TSAS que participan en el programa. Con ello se apoya a las entidades sociales en su situación de exigencia de información de acuerdo a la teoria institucional y de la dependencia de recursos.

A diciembre de 2018 en Juntos por el Empleo participan 1.451 entidades: más de 1.000 organizaciones sociales, 78 empresas y fundaciones empresariales y 17 administraciones públicas de ambito estatal o territorial y sus 22 soluciones para el empleo y el autoempleo se están utilizando por 911 entidades en 38 paises. Con estas soluciones se han realizado 453.195 diagnósticos de empleabilidad e impartido 33,2 millones de horas de formación a 449.361 personas. Ello ha facilitado la inserción laboral de 93.602 personas (Accenture, 2018).

Estas soluciones, que recogen el diagnóstico, análisis y mejora competencial y reporting para el empleo y el autoempleo, bases de datos, guías y plataformas online de formación y sistemas de medición del valor social, entre otras, están alojadas en una plataforma en la nube de Accenture y se ofrecen de forma gratuita a las entidades participantes, en forma de software como servicio.

El origen de algunas de estas soluciones es el conjunto de las bases de datos y los procedimientos de trabajo que utilizaban varias de las entidades del TSAS que se incorporaron a la plataforma y que compartían en los grupos colaborativos informales que tenían entre ellas. Basándose en esto, desde Fundación Accenture y con las entidades participantes en la gobernanza del proyecto, se ha producido un significativo desarrollo de las mismas estando planificado incluirse en el futuro próximo la realidad aumentada y la realidad virtual.

Las entidades del TSAS más involucradas en los inicios de Juntos por el Empleo y Fundación Accenture, en base a las necesidades detectadas en el mercado de trabajo para conseguir la inserción laboral de los beneficiarios, compartían y comparten unos objetivos colectivos para la mejora de los resultados de los programas de empleo, de los programas de autoempleo y para el apoyo a las entidades en su gestión y en la medición de impacto que demandan los financiadores.

En el marco del empleo la prioridad es formar en las necesidades reales del puesto de trabajo. Para ello, es necesario un buen mapeo de las competencias personales y técnicas para el mismo, un buen diseño de programas de formación para su mejora y la posibilidad de hacer prácticas no laborables.

En el marco del autoempleo o emprendimiento se busca optimizar y extender los programas de acompañamiento y desarrollar el espíritu emprendedor.

Así, alineadas con estos objetivos, las soluciones tecnológicas pueden agruparse también en tres tipos según su foco principal: soluciones para el empleo, soluciones para el autoempleo y soluciones transversales. 
Dentro de las soluciones para el empleo, además del Observatorio para el empleo ya mencionado, existe:

- la herramienta de diagnóstico social, competencial y profesional,

- la de competencias prelaborales, que incluye material de evaluación con un simulador on line y más de 75 cursos de formación en competencias, conocimientos digitales e idiomas

- la de reporting y

- varias guías que recogen metodología consensuada para la realización de prácticas en empresas, formaciones no regladas y actividades que mejoran la inserción laboral.

En las soluciones para el autoempleo, siendo el contenido muy similar a las de empleo, se incluyen soluciones específicas para el mentoring, la colaboración en red y la promoción de los microcréditos.

Por último, en las soluciones transversales se integran herramientas de gestión, de formación, de financiación y de impacto. Hay que hacer mención específica de la inclusión de las herramientas de gestión y de impacto. Con ello se facilita la información sobre el impacto social que los grupos de interés están demandando a las entidades del TSAS y el uso de una metodología estandarizada para su generación. Todo ello, facilitando la gestión de grandes cantidades de datos con el uso de soluciones tecnológicas.

Que una fundación empresarial financiadora de proyectos sociales, la Fundación Accenture, haya elegido como sistema para aportar fondos a las entidades del TSAS el ofrecerles gratuitamente herramientas tecnológicas de utilidad para su actividad, para su gestión y para su medición de impacto, apoyando con su aportación las exigencias de información que estas entidades tienen de otros financiadores y grupos de interés, es una nueva forma de abordar la solución de necesidades sociales y las demandas de reporting a las entidades del TSAS.

\section{Conclusiones}

El objetivo principal de este artículo es poner en relieve y explicar las razones por las que las entidades del TSAS tienen que responder a las exigencias de sus principales proveedores de recursos financieros respecto al tipo, formato y cantidad de información sobre sus actividades y resultados. La raíz de esta dependencia tiene explicación a través de la teoría institucional y la de la dependencia de los recursos, como argumentamos en el marco teórico que describe el artículo. 
Las presiones que las entidades del TSAS reciben de sus financiadores les obligan a satisfacer sus demandas de la forma que ellos requieren, lo que significa mejorar los métodos y herramientas con los que recopilar información y con los que reflejar y medir sus resultados económicos, sociales y medioambientales. Las tecnologías digitales son una herramienta fundamental para que las entidades del TSAS respondan eficientemente a las necesidades anteriores, tal como este estudio justifica.

El estudio del caso de Juntos por el Empleo como práctica que se está desarrollando para enfrentar estos requisitos por parte de las entidades del TSAS españolas nos ha permitido explorar las posibilidades de una respuesta colectiva entre entidades del TSAS, empresas y administraciones públicas para dar solución al problema planteado.

En un entorno en el que cada vez existe más presión a las entidades del TSAS por parte de sus grupos de interés para que generen información sobre su impacto social, es preciso identificar herramientas digitales y formas de trabajo con las mismas que apoyen el uso de la información de estas entidades para que sea de utilidad para su gestión y el cumplimiento de sus objetivos y para la realización de la evaluación de su impacto.

Por otro lado, el cumplimiento de los objetivos de las entidades del TSAS es cada vez más complicado dada la complejidad creciente de las necesidades sociales. Una nueva forma, colaborativa y estructurada, de abordar los problemas es necesaria; un enfoque de impacto colectivo.

La iniciativa de Juntos por el Empleo, funcionando como proyecto de impacto colectivo, ha facilitado y promovido el uso de la tecnologia para la mejora de eficiencia de las actividades de las entidades del TSAS y la sistematización de su gestión y medición de impacto simplificando los procesos de rendición de cuentas y dotándolos de transparencia.

En este artículo ofrecemos una solución al reto al que se enfrenta el TSAS de medir de manera representativa su actividad y realizar un seguimiento periódico de la misma para racionalizar su gestión y aumentar su eficacia, junto a la exigencia que le imponen los financiadores de que existan unos cauces de información adecuados con todos los agentes interesados que permitan alcanzar la transparencia informativa sobre la gestión de los recursos. La utilización de herramientas digitales que permiten sistematizar la recogida, gestión y transmisión de la información, mejorar los procesos internos, la rendición de cuentas y el control y la medición del impacto generado por las entidades es clave para afrontar el reto descrito.

En este sentido, y sobre la base del caso expuesto, sugerimos que los modelos de impacto colectivo inter-sectoriales constituyen una alternativa muy prometedora porque la colaboración entre todos los actores partícipes en el problema permite afrontarlo de forma holística y completa. Además, la generación y utilización de herramientas digitales puestas al servicio de las entidades del TSAS para mejorar los procesos dirigidos a dar solución a los complejos problemas, tanto sociales como de gestión, a 
los que se enfrentan; contribuye positivamente a la mejora de la eficiencia de estas entidades en el logro de sus objetivos.

Entendemos que el análisis que ofrecemos se encuentra en una fase inicial y que el caso que presentamos necesita de un desarrollo mayor. Así, en la siguiente fase del estudio nos dirigiremos a los distintos grupos de interés del proyecto descrito para realizarles entrevistas en profundidad que nos permitan entender las fortalezas y debilidades de este modelo intersectorial de impacto colectivo y plantear propuestas generales que permitan sistematizar este modelo para dar solución al problema de digitalización, monitorización y evaluación del impacto de las entidades del TSAS.

\section{Bibliografía}

ACCENTURE (2018): Impacto generado por juntos por el empleo. Disponible en: https://juntosporelempleo.cclearning.accenture.com/documents/10228/0/Impacto+Juntos+por+el+Empleo/23bfe31e73a3-4eab-9fdc-1f5615c1456a

ARVIDSON, M. \& LYON, F. (2014): "Social impact measurement and non-profit organisations: compliance, resistance, and promotion", VOLUNTAS: International Journal of Voluntary and Nonprofit Organizations, 25(4), 869-886.

BEBBINGTON, J., BROWN, J. \& FRAME, B. (2007): "Accounting technologies and sustainability assessment models", Ecological Economics, 61 (2-3), 224-236.

BIES, A.L. (2010): "Evolution of nonprofit self-regulation in Europe", Nonprofit and Voluntary Sector Quarterly, 39(6), 1057-1086.

COOPER, S.M. \& OWEN, D.L. (2007): "Corporate social reporting and stakeholder accountability: The missing link", Accounting, Organizations and Society, 32, 649-667.

COSTA, E. \& PESCI, C. (2016): "Social impact measurement: why do stakeholders matter?", Sustainability Accounting, Management and Policy Journal, 7(1), 99-124.

CRESPY, C.T. \& MILLER, V.V. (2011): "Sustainability Reporting: A Comparative Study of NGOs and MNCs", Corporate Social Responsibility and Environmental Management, 18, 275-284.

DHANANI, A. \& CONNOLLY, C. (2012): "Discharging not-for-profit accountability: UK charities and public discourse", Accounting, Auditing \& Accountability Journal, 25(7), 1140-1169.

DIMAGGIO, P.J. \& POWELL, W.W. (1983): "The iron cage revisited: Institutional isomorphism and collective rationality in organizational fields", American Sociological Review, 48, 147-160. 
EBRAHIM, A. (2003): "Accountability in practice: mechanisms for NGOs", World Development, 31(5), 813-829.

EBRAHIM, A. (2005): "Accountability myopia: Losing sight of organizational learning", Nonprofit and Voluntary Sector Quarterly, 34(1), 56-87.

EBRAHIM, A. \& WEISBAND, E. (2007): Global Accountabilities: Participation, Pluralism, and Public Ethics, Cambridge: Cambridge University Press.

EBRAHIM, A. \& RANGAN, V.K. (2014): "What impact? A framework for measuring the scale and scope of social performance", California Management Review, 56(3), 118-141.

ECKERD, A. \& MOULTON, S. (2011): "Heterogeneous roles and heterogeneous practices: Understanding the adoption and uses of nonprofit performance evaluations", American Journal of Evaluation, 32(1), 98-117.

EDWARDS, M. \& HULME D. (1995): Non-Governmental Organizations-Performance and Accountability: Beyond the Magic Bullet, Earthscan: London

EHNERT, I., PARSA, S., ROPER, I., WAGNER, M. \& MULLER-CAMEN, M. (2016): "Reporting on sustainability and HRM: A comparative study of sustainability reporting practices by the world's largest companies", The International Journal of Human Resource Management, 27(1), 88-108.

EISENHARDT, K.M. (1989): "Building Theories from Case Study Research", Academy of Management Review, 14 (4): 532-550.

FROELICH, K.A. (1999): "Diversification of Revenue Strategies: Evolving Resource Dependence in Nonprofit Organizations", Nonprofit and Voluntary Sector Quarterly, 28, 246-268.

GANDÍA, J.L. (2011): "Internet disclosure by nonprofit organizations: Empirical evidence of nongovernmental organizations for development in Spain", Nonprofit and Voluntary Sector Quarterly, 40 (1), 57-58.

GÁLVEZ RODRÍGUEZ, M., CABA PÉREZ, M. \& LÓPEZ GODOY, M. (2012): "Responsabilidad social y transparencia on-line de las ONG: análisis del caso español", CIRIEC-España, Revista de Economía Pública, Social y Cooperativa, 74, 207-238.

GIBELMAN, M. \& GELMAN, S.R. (2004): "A loss of credibility: Patterns of wrongdoing among nongovernmental organizations", Voluntas: International Journal of Voluntary and Nonprofit Organizations, 15(4), 355-381.

GOMES, C. (2009): "Computational sustainability: computational methods for a sustainable environment, economy, and society", The Bridge, 39(4): 5-13

HART, T.R. (2002): "EPhilanthropy: Using the internet to build support", International Journal of Nonprofit and Voluntary Sector Marketing, 7(4), 353-360. 
HELBING, D. (2012): "The futurICT knowledge accelerator towards a more resilient and sustainable future". In: Ball, P. (Ed), Why society is a complex matter, Springer, Berlin.

HIGGINS, C., STUBBS, W. \& MILNE, M. (2018): "Is sustainability reporting becoming institutionalised? The role of an issues-based field", Journal of Business Ethics, 147(2), 309-326.

JENSEN, O.A. \& SEELE, P. (2013): "An analysis of sovereign wealth and pension funds' ethical investment guidelines and their commitment thereto", SEELE, 3(3), 264-282.

KANIA, J. \& KRAMER, M. (2011): "Collective impact", Stanford Social Innovation Review, 9(1), 36-41.

KNEBEL, S. \& SEELE, P. (2015): "Quo vadis GRI? A (critical) assessment of GRI 3.1 A+ non-financial reports and implications for credibility and standardization", Corporate Communications: An International Journal, 20(2), 196-212.

KOLK, A. (2004): "A decade of sustainability reporting: developments and significance", International Journal of Environment and Sustainable Development, 3(1), 51-64.

LOCK, I. \& SEELE, P. (2016): "The credibility of CSR (corporate social responsibility) reports in Europe. Evidence from a quantitative content analysis in 11 countries", Journal of Cleaner Production, 122, 186-200.

MAAS, K., SCHALTEGGER, S. \& CRUTZEN, N. (2016): "Integrating corporate sustainability assessment, management accounting, control, and reporting", Journal of Cleaner Production, 136, 237248.

MEYER, J.W. \& ROWAN, B. (1977): "Institutionalized organizations: Formal structure as myth and ceremony", American Journal of Sociology, 83(2), 340-363.

MILNE, M.J., TREGIDGA, H.M. \& WALTON, S. (2009): "Words not actions! The ideological role of sustainable development reporting", Accounting, Auditing and Accountability Journal, 22(8), 12111257.

MOXHAM, C. (2010): "Help or hindrance? Examining the role of performance measurement in UK nonprofit organizations", Public Performance \& Management Review, 33(3), 342-354.

NICHOLLS, A. (2010): "The functions of performance measurement in social entrepreneurship: control, planning and accountability". In: K. Hockerts, J. Mair \& J. Robinson (Eds.), Values and opportunities in social entrepreneurship, Palgrave MacMillan: New York.

NGUYEN, L., SZKUDLAREK, B. \& SEYMOUR, R.G. (2015): "Social impact measurement in social enterprises: An interdependence perspective", Canadian Journal of Administrative Sciences, 32, 224-237.

PFEFFER, J. \& SALANCIK, G.R. (1978): The external control of organizations: A resource dependence perspective, New York: Harper \& Row Publishers.

POWER, M. (1999): The audit society: rituals of verifications, Oxford University Press: Oxford, UK. 
SAXTON, G. D. \& GUO, C. (2011): "Accountability Online: Understanding the Web-based Accountability Practices of Nonprofit Organizations", Nonprofit and Voluntary Sector Quarterly, 40 (2), 270-295.

SCHOLTE, J.A. (2004): "Civil society and democratically accountable global governance", Government and Opposition, 39(2): 211-233.

SCOTT, R. (1987): "The adolescence of institutional theory", Administrative Science Quarterly, 32, 493511.

SEELE, P. (2016): "Envisioning the digital sustainability panopticon: a thought experiment of how big data may help advancing sustainability in the digital age", Sustainability Science, 11(5), 845-854.

SINCLAIR, P. \& WALTON, J. (2003): "Environmental reporting within the forest and paper industry", Business Strategy and the Environment, 12, 326-337.

SUCHMAN, M.C. (1995): "Managing legitimacy: Strategic and institutional approaches", The Academy of Management Review, 20(3), 571.

VACCARO, A. \& MADSEN, P. (2009): "Corporate dynamic transparency: The new ICT-driven ethics?", Ethics and Information Technology, 11(2), 113.

VERBRUGGEN, S., CHRISTIAENS, J. \& MILIS, K. (2011): "Can resource dependence and coercive isomorphism explain nonprofit organizations' compliance with reporting standards?", Nonprofit and Voluntary Sector Quarterly, 40(1), 5-32.

VIGNEAU, L., HUMPHREYS, M. \& MOON, J. (2015): "How do firms comply with international sustainability standards? Processes and consequences of adopting the global reporting initiative", Journal of Business Ethics, 131(2), 469-486.

YIN, R.K. (1984): Case Study Research: Design and Methods, Applied social research Methods Series, Sage, Newbury Park, CA.

ZUCKER, L. (1987): "Institutional theories of organization”, Annual review of sociology, 13, 443-464. 
